Sir

\section{Reply to J Deane}

Thank you for giving me an opportunity to respond to the comments made by Mr Deane on the results of retinal detachment surgery performed by general ophthalmologists in a district general hospital. The author's point on the risk of macula-on detachment becoming macula-off before they are dealt with at the tertiary centre is a valid one, and to my knowledge has not been studied. I agree with the author in that if there is significant delay in patients with macula-on detachment reaching the regional centres, there may be a role for the ophthalmologists in the referring centres in their surgical management. However, I am not sure of the number of general ophthalmologists available with sufficient training and competence to take on retinal detachment surgery. Certainly, the newer generation of ophthalmologists without additional subspecialty training are unlikely to be able to perform such surgery.

The situation in our region is such that referral of patients with macula-on detachment is treated on an urgent basis and hence the risk of macula-on detachment becoming macula-off detachment before surgery is likely to be minimal. Any future college audit on retinal detachment surgery should be able to look at this aspect and provide us with an answer for this question and appropriate guidelines.

\section{S Dinakaran \\ Department of Ophthalmology, \\ Doncaster Royal Infirmary, \\ Armthorpe Road, \\ Doncaster DN2 5LT, UK \\ Correspondence: S Dinakaran \\ Tel: + 441302366666 ext 3050; \\ Fax: + 441302761208. \\ E-mail: sdinakaran@yahoo.com}

Eye (2005) 19, 691. doi:10.1038/sj.eye.6701551

Published online 4 March 2005

\section{Sir,}

Orbital cellulitis $v$ s allergic reaction to hyaluronidase as the cause of periorbital oedema

We read with interest the case report by Varma $e t a l^{1}$ regarding a case of presumed orbital cellulitis after peribulbar anaesthesia for cataract surgery. The patient presented 2 days postoperatively with periorbital oedema, redness, proptosis, conjunctival chemosis, and restriction of ocular movements on the operated eye, and improved following treatment with intravenous antibiotics.

We would like to report three cases, which presented 1-2 days postoperatively with the same apparent clinical appearance and outcome, but which we believe to represent a different diagnosis. All three cases presented with periorbital swelling within $48 \mathrm{~h}$ of surgery after uncomplicated peribulbar anaesthesia. Patients had mild (one or two lines) reduction of Snellen's visual acuity and no RAPD. There was no purulent discharge from the eye. All patients were apyrexial. Antibiotic treatment was not administered as the signs were attributed to an allergic reaction to hyaluronidase rather than infection, but the patients were closely observed.

As in Varma's case, each had a peribulbar anaesthesia, which included hyaluronidase. In all our cases, there had been previous exposure to hyaluronidase in the same or fellow eye, implying that sensitisation had taken place. Varma et al do not comment on whether their patient had had previous exposure to hyaluronidase, but she did have previous cataract surgery to the fellow eye, and may therefore have been sensitised.

The rapid onset of signs in the absence of pyrexia and with negative blood and tissue cultures in the case noted by Varma et al, lend support to the possibility that this case may also have been allergic rather than infection. Allergy to hyaluronidase is a recognised complication. In cases noted by Kirby et $a l_{,}^{2}$ Minning ${ }^{3}$ and Taylor $e t$ al ${ }^{4}{ }^{4}$ type I allergic reaction to hyaluronidase during surgery was confirmed later with skin-patch testing. A feature of these reactions was marked periorbital oedema.

The possiblity of allergic reaction to hyaluronidase should be considered when a patient presents with a rapid onset of signs, especially if the patient has had previous exposure to hyaluronidase.

\section{References}

1 Varma D, Metcalfe TW. Orbital cellulitis after peribulbar anaesthesia for cataract surgery. Eye 2003; 17: 105-107.

2 Kirby B, Butt A, Morrison AM, Beck MH. Type I allergic reaction to hyaluronidase during ophthalmic surgery. Contact Dermatitis 2001; 44(1): 52

3 Minning CA Jr. Hyaluronidase allergy simulating expulsive choroidal hemorrhage. Arch Ophthal 1994; 112(5): 585-586.

4 Taylor IS, Pollowitz JA. A little known phenomenon: allergic reaction to hyaluronidase. Ophthalmology 1984; 91: 1003.

\section{Z Youssef, PM Pennefather and MT Watts}

Arrowe Park Hospital, Upton

Wirral, CH49 5PE, UK 